\title{
Zum Beweis der trajektoriellen Natur der Plasmastrahlungen.
}

\author{
Ein Beitrag zur Mechanik der Mitose. \\ Von \\ Eriedrich Reinke. \\ Aus dem anatomischen Institut in Rostock.) \\ Mit 11 Figuren im Text. \\ Eingegangen am 25. Oktober 1899.
}

Die Mechanik der Zelltheilung und die mit derselben eng verknüpfte Plasmastruktur erregen seit Jahren das größte Interesse der biologischen Forschung und haben demgemäß eine Fluth von theoretischen Erörterungen hervorgerufen, welche in den Referaten von W. Flemming und F. Meves (Ergebnisse der Anatomie und Entwickelungsgeschichte von Fr. Merkel und R. Bonnet 1897) leicht nachgesehen werden können. Außerdem ist für diese Fragen von besonderer Wichtigkeit die größere Arbeit von WrLHelm His, Über Zellen and Syncytienbildung (Bd. XXIV der Abhandlungen der K. S. Gesellschaft der Wissenschaften).

Eine Hauptrage bei der Zelltheilung ist die, welche Bedeutung haben die Strahlungen nebst der Spindel im Protoplasma der Zelle?

Seitdem Fol (Die erste Entwickelung des Geryonideneis, Jenaische Zeitschr. Bd. VII. pag. 176) im Jahre 1873 die plasmatischen Doppelsterne mit magnetischen Kraftlinien verglichen hat und die Mittelpunkte der Sterne als Anziehungscentren bezeichnete, haben die Ahnlichkeit dieser Dinge zahlreiche Autoren wie Strassburger, Hennegux, van Beneden, Errera und namentlich Ziegler und Gallardo hervorgehoben, während BÜTSCHLI, von ERLANGER und RHUMBLER diese charakteristischen Figuren auf Zugkräfte zuriickführen. 
Doch hat es keineswegs an Widerspruch gefehlt. Namentlich ist Meves 1. c. gegen diese Anschanungen zu Felde gezogen, indem er ausfuhrt, dass in der Thatsache, dass die von den Centralkörpern ausgehenden Polstrahlen sich häufig in ausgedehnter Weise durchkreuzen, der Beweis liege, dass die Erscheinungen nicht vergleichbar wären, da eine derartige Durchkreuzung bei Kraftlinien, welcher Art sie auch sein mögen, ausgeschlossen sein soll. Ferner führt Meves weiter aus, »dass, wenn von zwei in einer Zelle vorhandenen Centren gleiche Wirkungen von der Art, wie Bürschli sie annimmt, auf das Cytoplasma ausgeiubt werden, niemals eine spindelartige Figur zwisehen den Centren entstehen kann. Wenn Bürschli thatsächlich, wie er beschreibt, Spindelfiguren beobachtet hat, welche in warm aufgestrichenen Gelatineölschäumen zwischen zwei gleich großen Luftblasen beim Erkalten anftreten, so muss diese Erscheinung in anderer Weise erklärt werden als durch einen allseitig zum Centrum der Blase gerichteten Zug. Denn in diesem Falle kann die Anordnung der Strahlen nur eine solche sein, wie diejenige von Kraftlinien, wie sie von zwei Kraftcentren mit gleichen Potentialen und gleichen Vorzeichen ausgehen *.

Diese Meves'sche Kritik ist keine gliickliche. Schon Rhumbler ( Die Mechanik der Zelldurchschnürung nach Meves' und nach meiner Auffassung«, Archiv f. Entwickelungsmech. VII. pag. 534) hat eine Widerlegung gebracht, der man im Allgemeinen zustimmen kann. Indem derselbe an einem Gummimodell nachweist, dass durch einen allseitig zum Centrum gerichteten Zug, beim Vorhandensein zwei solcher Centren, stets Spindelfiguren zwischen den Centren entstehen und dass durch Ungleichzeitigkeiten der beiderseitigen Zugwirkungen, welche etwa durch zufällige Ungleichheiten und Störungen eintreten müssen, eine Durchkreuzung der Strahlen eintreten muss.

Schon eine einfache Überlegung zeigt sofort, dass wenn zwei Luftblasen in einer erkaltenden Ölgelatine sich verkleinern, der Zug, den sie beide auf die zwischen ihnen liegende Masse ausuben, in entgegengesetzter Richtung geschieht, den Zugkräften also auch entgegengesetztes Vorzeichen zukommt. Was sodann die Durchkreuzung der Strahlen angeht, so erklärt sie sich thatsächlich durch ungleichzeitige Wirkung der Kraftcentren aufs beste. Es braucht nur das eine Centrum dem anderen in der Wirkung etwas voraus zu sein oder die Wirkung derselben braucht nur zeitweise intermittirend zu geschehen, so werden sich die Strahlen nicht mehr in der Gleichgewichtsebene (neutralen Ebene oder Symmetrieebene) vereinigen, 
Fig. 1.

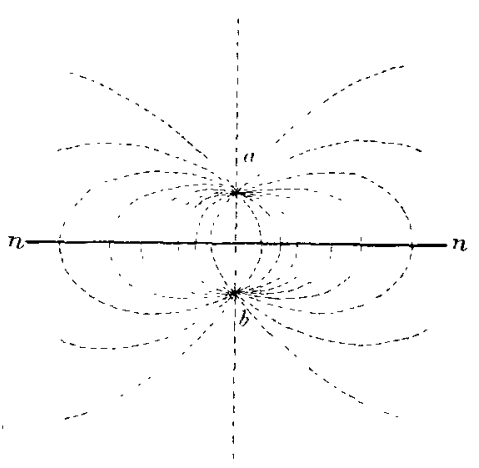

Fig. 2.

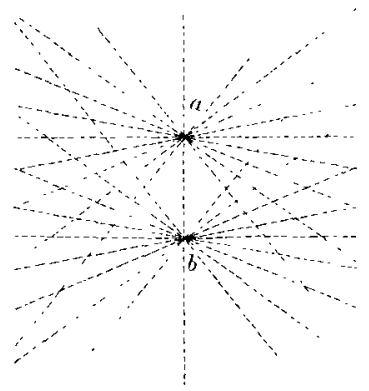

Fig. 3.

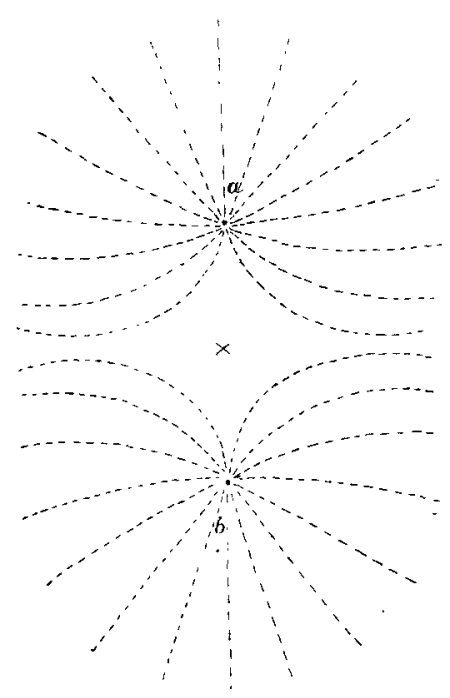

sondern die beiderseitigen Strahlen werden sich durchkreuzen und zrar unter um so stumpferen Winkeln, je näher sie an der Verbindungslinie beider Centren liegen.

Um den Leser sogleich iiber diese Dinge zu orientiren, gebe ich hier einige Diagramme elektrischer Kraftlinien (dies sind Richtungeu stärkster Übertragung einer Kraft ron Theil zu Theil in einem Körper $=$ Trajektorien) und zwar erstens in Fig. 1 für die Kraftlinien zweier elektrisirter Punkte mit den Ladungen + 10 und - 10, bei gleichzeitiger Wirkung. In Fig. 2 sieht man die Kraftlinien derselben Punkte (gleiche Ladung, ungleiches Vorzeichen;, welche ungleichzeitig wirken. Beide nach gauz genauen Berechnungen des beriuhmten Physikers JAMLs CLARK Maxwell Die Elektricität in elementarer Behandlung, herausgegeben von W. GarNeT, ubersetzt ron $\mathrm{L}$. Grätz: Fig. 3 endlich stellt die Kraftlinien dar zweier Centren mit gleich stark und gleichzeitig wirkender Ladung, aber gleichen Torzeichen, cine Figur, welche ich den Arbeiten Gallardo's entnehme, ahnlich wie sie auch Meves in seinem Referat giebt.

Fig. 4 giebt zum Vergleich die Strahlungen zweier Seeigeleier in Furchung, $a$ mit Spindelbildung, $b$ mit Durchkreuzung der Strahlen und dem zwischen den Centren noch mit Membran rersehenem Kern im Knäuelstadium. Stadium $b$ ("Befruchtungsstrablung*) geht dem Stadinm $a$ 
() Theilungsstrahlung «) voraus. Wie BütschLx jüngst bemerkt, finden sich derartige Bilder vielfach in der großen Arbeit von CarNor und LEBRUs. Besonders schön finden sie sich auch bei KostaneckI, Rhumbler nud vielen Anderen. Alle neueren Arbeiten über sich furchende Eier zeigen solche Bilder in großer Menge und dabei ist es besonders nach BoverI etwas ganz Gewöhnliches, dass die einen Strahlen den anderen etwas voraus sind, was auf eine etwas ungleichzeitige Wirkung der Kraftcentren schließen lässt.

Sprechen diese seltenen Übereinstimmungen der Kraftliniendiagramme mit den Figuren in den Zellen schon durchaus zu Gunsten der FoL'schen Auffassung, so kann ich noch einen weiteren Beleg

Fig. 4.
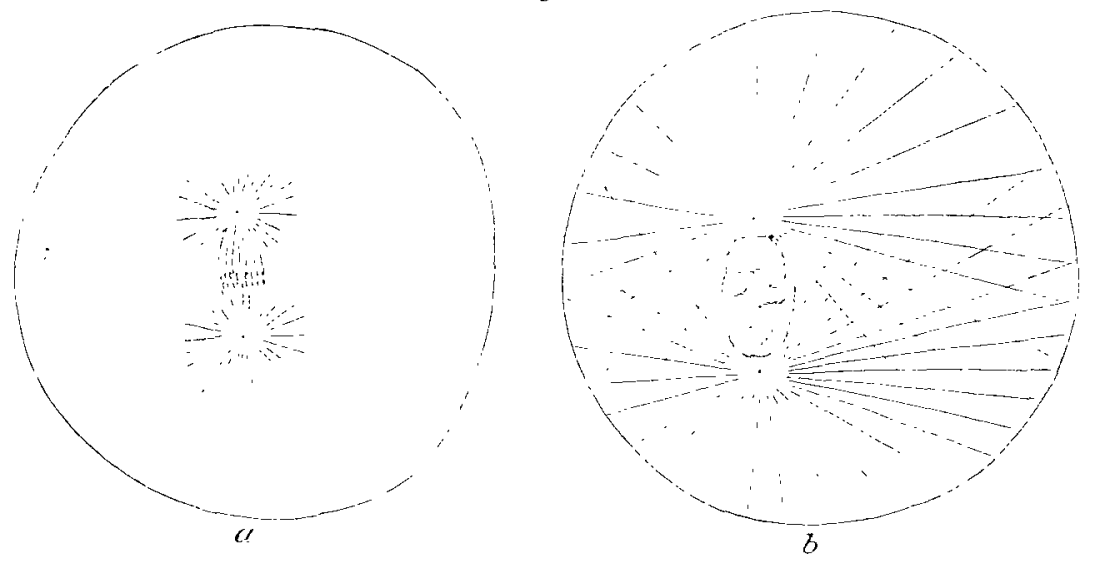

liefern, der, wie mir scheint, außerordentlich für die trajektorielle Natur der Zellstrahlungen spricht.

Die angefuihrten Diagramme stellen stets Kraftlinien dar, die von gleich starken Centren gebildet werden. Es muss danach angenommen werden, dass auch die beiden Centren der Zellen gleich groß sind. Wie wenn man weiter variiren könnte und Figuren zum Vergleich heranzöge, deren Centren ungleich stark sind? Ich bin thatsächlich in der angenehmen Lage dies thun zu können.

In Fig. 5 und Fig. 6 gebe ich, wieder nach Maxwecl, die Diagramme zweier Kräftepaare, von denen sich das erste in Fig. 5 verhält wie $4: 1$, während das zweite in Fig. 6 sich verhält wie $5: 3$. Das Charakteristische in diesen Figuren gegenüber der Fig. 1 sind folgende drei Punkte:

Erstens: Die Strahlung ist am stärkeren Centrum (a) viel bedeutender als am schwächeren Centrum $(b)$. 
Zweitens: Die Spindel erscheint am stärkeren Centrum (a) mehr verjungt, am schwächeren Centrum $(b)$ mehr verbreitert, sie ist also inäqual-bipolar symmetrisch gebaut.

Drittens: Die Gleichgewichtsfläche (symmetrische oder neutrale Fläche $n n$ ), in welcher sich die Linien der Spindelhälften vereinigen, ist dem schwächeren Centrum zu konkav gekrümmt. Sie bildet eine Kugelschale, deren Konkavität dem schwächeren Centrum zugewandt ist. Diese charakteristische Krümmung nimmt zu mit der Größendifferenz der Centren. Sie ist in Fig. 5, wo sich die Kräfte verhalten wie $4: 1$, größer als in Fig. 6 , wo sich dieselben wie $5: 3$ verhalten. Außerdem ist die Länge des Abstandes des stärkeren Centrum $a$ von

Fig. 5 .

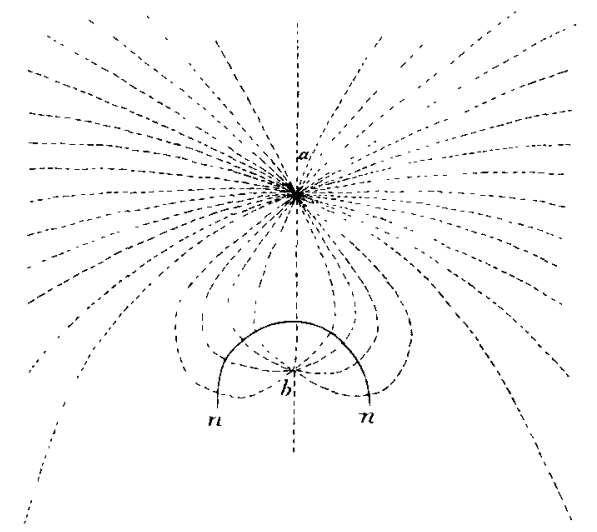

Fig. 6.

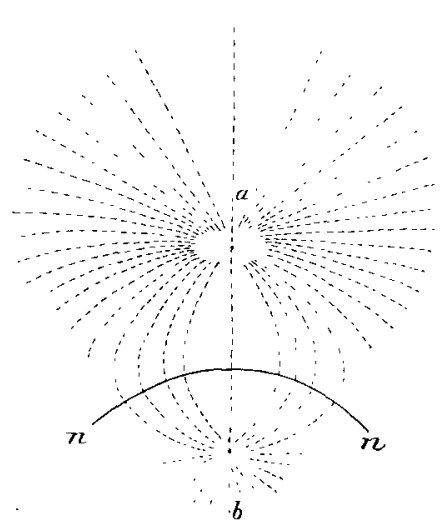

der Gleichgewichtsfläche $n n$ bedentend größer als die Länge des Abstandes des schwächeren Centrums $b$ von $n n$. Mit anderen Worten: zum stärkeren Kräftecentrum gehört ein größerer Spindeltheil als zum schwächeren Kräftecentrum.

In Fig. 7 gebe ich die Abbildung einer Mitose aus einem Flächenpräparat (kein Schnitt!) des Bindegewebes einer rasch wachsenden Salamanderlarve. Es ist eine ganz flache Zelle, die abgeplattete Spindel liegt genau parallel mit der Ebene des Objektträgers, so dass die beiden Centren nebst Strahlungen bei ein und derselben Einstellung deutlich zu sehen sind. Fixirung und Färbung des Präparates erscheinen tadellos. Die Figur ist mit dem Zesss'schen Zeichenapparat, Oc. II, Apochr. 2,0 mm von E. HarTNack, Höhe des Objekttisches, gezeichnet. Diese Art Zellen gehören zu den Syncytien im Sinne von W. HIs, nämlich Plasmagebilden, in denen die einzehnen Gebiete, wenigstens 
während der Mitose, unterscheidbar aber nicht abgegrenzt sind, wie wir sie im Bindegewebe der Salamanderlarve so vielfach finden. Die Mitose selbst ist eine Äquatorialplatte oder Mesophase, welche dadurch charakterisirt wird, dass die Chromosomen mit ihren Scheiteln (noch als Doppelschleifen) sich in den $\ddot{A} q u a t o r$ der Spindel einstellen. Es ist dies das Höhestadium der Mitose. Dasselbe ist von beträchtlicher Zeitdauer und stellt gewissermaßen einen Ruhepunkt in der Flucht der mitotischen Erscheinung dar.

Das vorliegende Präparat zeigt aber bedeutende und sehr wichtige Unterschiede von einer gewöhnlichen Mitose in der Mesophase. Und zwar in drei Punkten:

Erstens sind die Polstrahlungen ganz ungleich entwickelt, zu dem einen Centralkörperchen gehört eine beträchtliche, schön und regelmäßig ausgebildete Strahlung, am anderen Centralkörperchen finden sich nur wenige kurze Strahlen, gleichsam nur die Andentung einer Polstrahlung.

Zweitens: Die zur größeren Strahlung gehörige Spindelhälfte ist länger ausgezogen und läuft spitzer zu, während die zur schwäeheren Strahlung gehörige SpinFig. 7 .

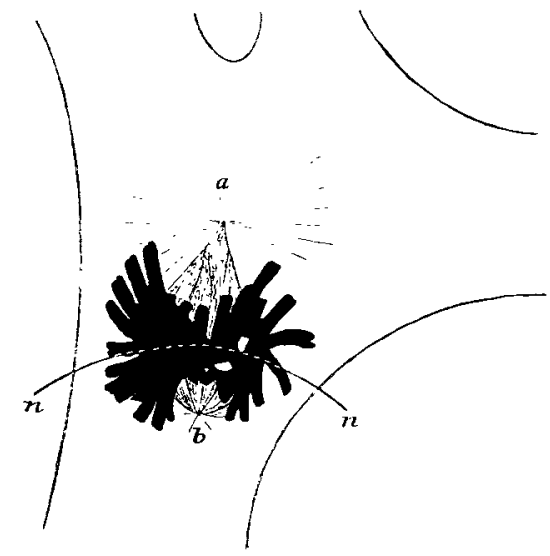
delhälfte breiter und kürzer gestaltet ist. Wir haben es demnach mit einer inäqual-bipolaren symmetrischen Figur zu thun.

Drittens: Der Ring der Chromosomen ist inäqual gestellt, indem er von den Centralkörperchen mit der größeren Strahlung abgeriickt erscheint. Dabei sind die Schleifen der Chromosomen so viel stärker gegen das Centralkörperchen $b$ gebogen, dass eine durch den ganzen Chromosomenkomplex hindurchgelegte Symmetriefläche nicht wie sonst eine Ebene, sondern eine gegen $b$ konkar gekrümmte Kugelfläche darstellt.

Es genügt einen vergleichenden Blick auf Fig. 6 des von MAXwELL berechneten Diagramms zweier elektrischen Centren von der Ladung $a=+5$ und $b=-3$, zu thun, um sogleich zu bemerken, dass beide Figuren eine verbliffende Ähnlichkeit haben, die noch auffallender ist, wenn man Fig. 6 durehgepaust auf Fig. legt, da 
sich dann die äußeren Spindeltrajektorien der Fig. 6 mit dem Umriss des Chromosomenkomplexes im Wesentlichen decken, die inneren Trajektorien aber mit denen der Spindel. Außerdem ist die Krummung der Gleichgewichtsfläche die gleiche. Man wird danach mit Wahrscheinlichkeit sagen können, dass auch in Fig. 7, also im Präparat, die Kräfte der Centren sich verhalten wie 5:3.

Die Sache lässt sich noch weiter treiben. Es fragt sich wie wird diese inäquale Mitose weiter verlanfen. Dies lässt sich nach meinen Präparaten dahin beantworten, dass

Fig. 8.

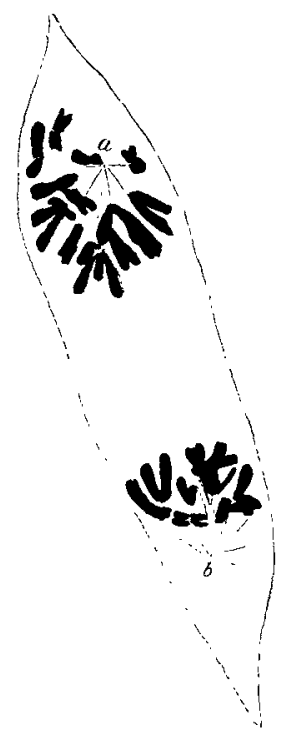
es wahrscheinlich zu einer inäqualen Theilung der Chromosomen kommt und zwar ohne die Trennung der Doppelschleifen. Wenigstens finden sich Bilder wie Fig. 8. Es sind dies zwei getrennte Tochterchromosomgruppen. Zum einen Centralkörperchen gehören 15 Doppelschleifen, zum anderen nur 9 Doppelschleifen. Es hat sich hier also der Kern mit seinen ursprünglichen 24 Doppelschleifen inäqual getheilt. $15: 9$ ist aber gleich $5: 3$, also genau wie vorhin in Fig. 6 und Fig. 7. Diese Chromosomen lassen sich im Präparat noch viel deutlicher zählen als in der Zeichnung, wo sie theilweise sich decken. Auch diese Zeichnung ist mit dem Zeichenapparat bei derselben Vergrößerung wie Fig. 7 angefertigt. Der Umstand, dass die Doppelschleifen, ohne sich zu spalten, einfach im Verhältnis von 5:3 aus einander gerückt sind, ist sehr merkwürdig. Auffällig ist, dass einige Chromosomen sehr kurz sind und in der Mitte wie durchgeschniurt erscheinen. Im Präparat lassen sich alle deutlich als Doppelfäden erkennen.

Mir sind derartige Abweichungen der Mitose aus der Litteratur nicht bekannt. Die heterotypischen Formen im Hoden sind wesentlich anderer Natur, da hier die Centren stets gleich zu sein scheinen und die Doppelfäden der Tochterchromosomen auf eine zweite Längstheilung, nicht auf ein Unterbleiben der Längstheilung bezogen werden. Der Gedanke, dass es sich in Fig. 8 um eine Zelle mit zwei Kernen in Mitose handle, wie ich zuerst annahm, ist auszuschließen, da von Spindeln und zweiten, etwa verdeckten Centralkörperchen keine Rede sein kann. ZIEGLER, Experimentelle Studien ïber die Zelltheilung. III. Archiv f. Entwickelungsmech. Bd. VII. pag. 47, stellt die Hypothese auf, dass bei der Mikromerenbildung eine Ungleichheit der Centren im Spiele sei. Ein Vergleich der betreffenden Figuren mit meinen Ałbilàungen spricht nicht dafür. Ebensowenig findet sich bei der Bildung der Richtungskërper etwas Derartiges. 
Fassen wir unsere Resultate zusammen so können wir folgende Sätze aufstellen.

1. Die Plasmastrahlungen der Mitose 'decken sich mit den Trajektorien elektrischer Kräftepaare aufs genaueste, auch in den Varietäten und es muss desshalb eine trajektorielle Natur der Strahlungen anerkannt werden.

2. Während der Mesophase liegen die Scheitel der Chromosomen in der *Gleichgewichtsfläche«.

3. Die Kräftecentren der regelrechten Mitose sind gleich stark aber mit entgegengesetzten Vorzeichen. Sie können gleichzeitig oder ungleichzeitig wirken.

4. Es giebt in seltenen Fällen Mitosen mit ungleich starken Kräftecentren und entgegengesetzten Vorzeichen. In diesem Falle bildet die »Gleichgewichtsfläche eine Kugelsehale.

Diese Stärkedifferenz der Kräftecentren führt zu keiner regelrechten sondern zu einer inäqualen Theilung der Chromosomen lohne Längsspaltung), deren Zahl direkt proportional der Differenz der Kräftecentren ist.

Wenn ich für den Trajektoriencharakter der Plasmastrahlungen eintrete, so scheint es nicht unangemessen einige Worte über die Entstehung solcher trajektoriellen Strukturen hier anzufügen. Unter Trajektorien verstehen wir nach W. Roux, »Beiträge zur Morphologie der funktionellen Anpassung " in: Gesammelte Abhandlungen. Bd. I.

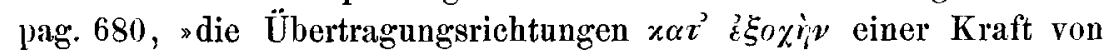
Theil zu Theil in einem Körper«. Bei elektrischen Kräften bezeichnen wir diese Kraftlinien speciell als Induktionslinien (FAradar). Solche Trajektorien brauchen aber keineswegs nur durch magnetische oder elektrische Kräfte hervorgebracht zu werden, sondern wie bekamnt erzeugen Spannkräfte in einem Körper ebenfalls Kraftlinien. Nach den Arten der Spannungen können wir z. B. Zugtrajektorien, Drucktrajektorien und Schubtrajektorien unterscheiden. Nach den Untersuchungen von G. H. MeYer, J. WoLfF und namentlich W. Roux stellt die Architektur der Spongiosa der ausgewachsenen Knochen, sowohl im normalen, wie nach längerer Zeit auch im anormalen Zustand solehe Trajektorienkonstruktionen dar, gerade wie sie in der konstruktiven Technik verwandt werden. Es ist selbstverständlich, dass diese Druck- und Zugtrajektorien selbst weder ziehen noch drücken. Der Sinn ihrer Anwendung bei organischen Substanzen wie in der Technik ist der, dass ein Körper, welcher Druck-oder Zugkräften Widerstand 
leisten soll (also auf Kompression nnd Dehnung in Anspruch genommen werden soll), bei Einhaltung einer gewissen änßeren Gestalt und Umfang in haushälterischer Weise mit einem Minimum von Material gebaut werde. Diese Konstruktion kann also nur dort angewandt werden, wo man mit einer geringeren Tragfähigkeit ans-

Fig. 9.

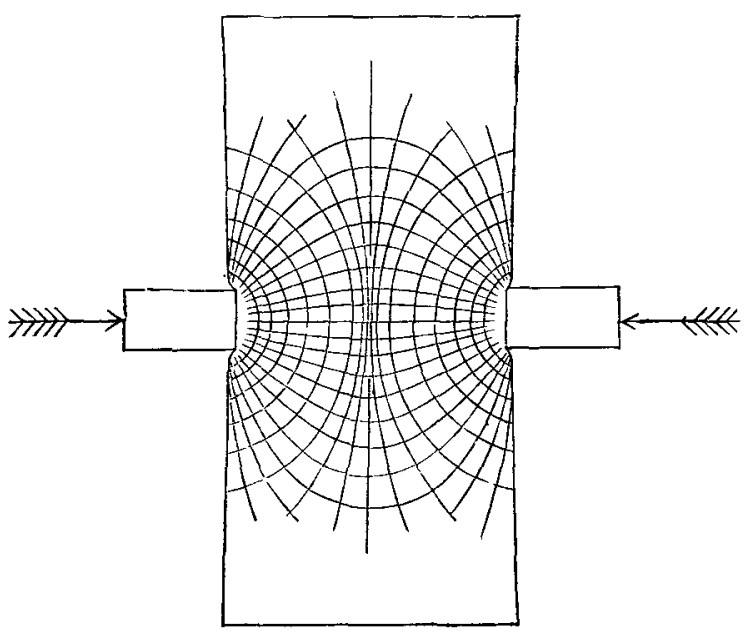

kommt, als sie eine kompakte Masse von gleichem Umfange gewährt. Wir verfahren bei den einfachsten Gebrauchsgegenständen ebenso. Wir stellen einen Tisch, einen Stuhl auf nur vier senkrechte Beine, indem diese den Anforderungen ebenso genügen als wie ein massiver Block, der eine Verschwendung von Tragfestigkeit darstellen wiirde. Es kommt der Natur, wie den menschlichen Konstruktionen stets darauf an, die nöthige Widerstandskraft mit dem Minimum an Material oder mit dem verwandten Material das Maximum an Leistung hervorzubringen. Es handelt sich nach W. Roux um

Fig. 10.

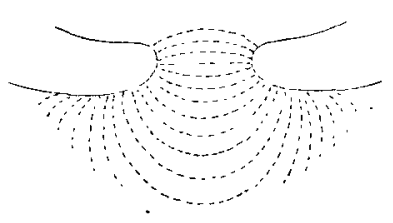
ein Minimum-Maximum-Princip der Konstruktion. (W. Roux, Funktionelle Gestalt und Struktur der Delphinflosse. Gesammelte AbhandI. Bd. I. pag. 508.)

Dabei sind die Figuren, welche man bei Anwendung von gleichen Zugoder Druckkräften erhält, sehr ähnlich den Kraftlinien zweier elektrischer Kräfte. Als Beispiel diene Fig. 9 und Fig. 10 nach W. Roux, wo im ersten Falle die pressenden Kräfte vollkommen entgegengesetzte Richtung: haben, im zweiten Falle dagegen dieselben etwas schräg gegen einander stehen. Die Linien der Spindel stellen die primären Drucktrajektorien, die darauf senkrechten die sekundären Zugtrajektorien dar; wobei zu bemerken ist, dass der sekundäre Zug nur $1 / 3-1 / 2$ der 
Größe des primären Druckes beträgt. Die Zugtrajektorien stehen stets rechtwinklig auf den Drucktrajektorien.

In den Knochen ist diese trajektorielle Struktur nun so ausgeführt, dass die Richtung der Maschen der Spongiosa diesen beiden Richtungen des stärksten Druckes und Zuges entspricht und diese Struktur entwickelt sich nach Roux in der Weise, dass der vou außen wirkende Druck und Zug, sei es das Körpergewicht, sei es der Muskeldruck einen funktionellen Reiz auf die lebende Knochensubstanz ausüben, die dort, wo dieser Reiz am stärksten einwirkt, sich erhält, während sie dort, wo dieser Reiz nicht wirkt, schwindet, dazu kommt, dass, wenn die den Trajektorien entsprechenden Richtungen genügend fest durch Knochensubstanz ausgebildet sind, sie den anderen Richtungen den Druck entziehen (W. Roux, Gesammelte Werke. Bd. I. pag. 356).

Übertragen wir diese Erfahrungen an Knochen auf das Plasma der Zelle, so werden wir zu der Anschauung gelangen, dass die Kräftewirkungen der Centralkörperchen (welche ja zunächst mit den Spermien in die Eizelle gelangt sind und sich dann durch Zweitheilung auf alle Gewebszellen fortgepflanzt haben auf das Plasma einen funktionellen Reiz ausuben, welcher dasselbe zwingt in bestimmter Richtung, nämlich der Richtung stärkster Übertragung (also den Trajektorien) sich anzuordnen. So wird die von dem Kräftepaar beherrschte morphoplasmatische Substanz gezwungen sich in die Gestalt der Trajektorien umzuformen, während dazwischen nur der Zellsaft, das Hyaloplasma bleibt.

Über die Art der Kräfte wissen wir noch nichts Sicheres. An und für sich, nach der Gestalt der Trajektorien, könnten es ebenso gut Zug- wie Druckkräfte oder elektrische und magnetische Kräfte sein. Allein da die Wirkung von so winzigen Körpern, wie die Centralkörperchen sind, ausgehen, so ist kaum an Zugkräfte im Bürschli'schen Sinne zu denken, noch weniger an Druckkräfte, dagegen scheint man zunächst mit elektrischen Kräften am weitesten zu kommen. Der eine stichhaltige Einwand ist wohl der, dass, da das Kräftepaar sicher entgegengesetztes Vorzeichen haben muss, nicht so ohne Weiteres ein Auseinanderrücken der Centren vorstellbar wird. Betrachtet man aber die Sache so, dass die lebende Substanz, durch das Kräftepaar in Reizzustand versetzt, sich zwischen diese einschiebend zur Spindel anwächst, so kann ich auch hierin keine Schwierigkeit weiter finden. Es würde das dann in der That eine Art von Expansionsvorgang sein. Allein ein weiterer Befund, der 
elektrische und magnetische Kräfte ausschließt, ist das Vorkommen von tripolaren und multipolaren Mitosen, da hier die Entstehung der Spindeln durch nur zwei Arten der Elektricität nicht erklärbar wäre. Es kommen aber auf noch ganz andere Weise trajektorielle Figuren zu Stande, z. B. bei der Krystallisation gewisser Chemosen. Vorzügliche Trajektorien kann man bei der Krystallisation der Karbolsäure beobachten, wobei man leicht pluripolare Spindeln erhält. Die Anregung zu diesen Beobachtungen bekam ich bei Nachahmung der Roux'schen Versuche der »kiunstlichen Kopulation«. Das Allgemeinvergleichbare an diesen Figuren scheint mir die Trajektoriennatur derselben zu sein, während daraus iuber die Art der Centralkörperkrïfte selbst natürlich nichts Genaneres zu eruiren ist.

Auf diese Weise stellt sich die Bildung der Polstrahlungen und der Spindel als ein Vorgang heraus, der lediglich durch die Centralkörperchen und das Morphoplasma des Zellleibes inklusive des plasmatischen Liningeriistes des Kernes, also des Cyto- und Karyoplasmas, hervorgebracht wird, ganz unabhängig vom Chromatin des Kernes. Auch olne das Chromatin würden wir vermuthlich dieselben Erscheinungen haben, wie das sich anch aus den wichtigen neueren Beobachtungen Boveri's und ZiEGLER's ergiebt.

Es drängt 'sich alsbald die weitere Frage auf, wie kommt es, dass die Schleifen der Chromosomen mit ilıren Scheiteln gerade in der »Gleichgewichtsfläche" verankert werden, ein Vorgang, der sowohl bei gleich starken als auch ungleich starken Kräftepaaren eintritt. Gerade letztere, in Fig. 7 , scheint mir verständlich zu machen, dass die Clromosomen, welche ja im Morphoplasma (speciell im Linin des Kernes) eingebettet liegen, durch die Fasern, welche der von den Centralkörperchen gesetzte Reiz herausbildete, ohne Weiteres mit diesen verbunden werden. Die Länge dieser Fäden wird aber, wie Fig. 7 zeigt, durch die Stairke der Centren bedingt. Mit dem Moment, wo die Centrilspindel voll ausgebildet ist, werden demnach auch die Chromosomen die Gleichgewichtslage annehmon. Jedes Lageverhältnis außerhalb der Gleichgewichtslage wird auf die lebende Fädenverbindung zwischen Chromosom und Centralkörperchen einen Zug ansüben, dem die lebende Substanz der Fäden so schnell wie möglich zu entgehen sucken wird. Wie dies geschehen mag siehe weiter unten.

Das ganze protoplasmatisch trajektorielle Gebilde stellt ăber unter Anderem eine Art von Zellskelet dar', welches durch seine Druckfestigkeit wohl geeignet erscheint dem mitotischen Druck ein Gegen- 
gewicht zu bieten, das sofort aufhört beim Erlöschen der Kraftwirkung der Centren. Zwar wird das Morphoplasma seine durch die Centren erhaltene Gestaltung noch einige Zeit beibehaltén, aber die Fäden werden jetzt durch den mitotischen Druck aus einander gedrängt werden, ohne wesentlichen Widerstand leisten zu können, wie wir das nach Theilung der Mutterchromosomen in die Tochterchromosomen bei fast jeder Zellenart beobachten können. Hier sehen wir denn auch die viel schwächeren queren Verbindungen der Spindelfaden, welche diese offenbar in senkrechter Richtung verknüpft haben und so die statische Konstruktion vervollständigten; wie das geschieht mag dahingestellt bleiben. Ich gebe hierzu eine Abbildung solcher Fäden nach Meves in Fig. 11. Ich bemerkte schon, dass eine solche statisch Fig. 11. trajektorielle Konstruktion, wie wir sie offenbar in der Mesophase vor uns haben, an sich nicht geeignet ist zu drücken oder zu ziehen und ich bin daher auch der Meinung, dass der Transport der Chromosomen mit dieser Striktur an sich nichts zu thun hat. Nun ist aber diese trajektorielle Struktur zugleich eine plasmatisch lebendige und was das lebende Protoplasma Alles zu leisten vermas, das wird der am besten ersehen künnen, der einmal genau das Protoplasma einer lebenden Pflanzenzelle, z. B. die Zellen der Haare an Knospen und Blättern des Kürbis oder eines anderen günstigen Objekts studirt hat.

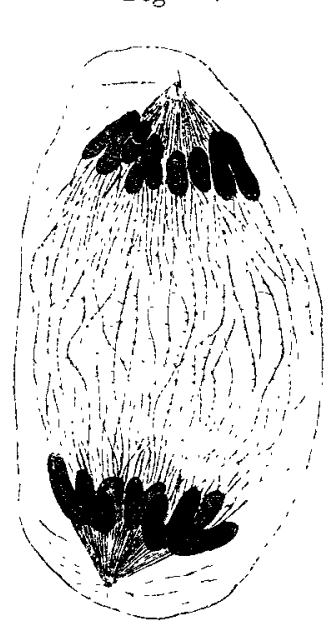

M. ILenenhais hat kürzlich in einem Aufsatz: "Einiges über die sogenannten Protoplasmaströmungen * (Sitzungsberichte der pliys.medicinischen Gesellschaft zu Würzburg), darüber eingehender berichtet. Ich habe dieses prachtvolle Objekt mir ebenfalls genauer angesehen. Jede dieser langgestreckten Zellen zeigt einen protoplasmatischen Wandbelag, den sogenannten Primordialschlauch, dessen Inhalt aus Flissigkeit besteht. Im Inneren findet sich meistens ein axialer dickerer Protoplasmastrang, in dem der Kcrn eingebettet ist, von dem aus zahlreiche dickere, bandartige oder fadenförmige, oft verästelte Protoplasmastränge, den Binnenraum durehziehend, am Wandbelag endigen.

Auf zwei Phänomene will ich hier nur linweisen, welche mit der 
Bewegung der Chromosomen vergleichbar sind. Einmal sieht man sehr häufig »wie feinere oder gröbere Protoplasmaklumpen (im Gegensatz zu der ebenfalls sichtbaren Körnchenströmung) an den Strängen entlang gleiten, oder wie bald mehr bald weniger umfängliche Plasmamassen in der Bahn eines relativ dünnen Stranges sich vorwärts schieben oder nach einer bestimmten Gegend hin sich zuriekziehen, oder wie ein feiner Strang vor unseren Augen aus benachbarten Gegenden große Massen lebenden Materials aufnimmt, um auf das Vielfache seines Durchmessers anzuschwellen (M. Heidenuain). Das sind offenbar höchst komplicirte Processe, die schwerlich sich mechanisch erklären lassen, deren Vorkommen aber, wie ich bestätigen kann, etwas ganz Gewöhnliches ist. Ferner habe ich des öftern beobachten können, dass ein ganzer verästelter Protoplasmafaden, der einerseits mit dem axialen Protoplasma, andererseits mit dem protoplasmatischen Wandbelag in Verbindung stand, als Ganzes durch den Binnenraum der Zelle sich ein beträchtliches Ende, nämlich von einem Ende der Zelle zum anderen, fortbewegte, ohne seine Gestalt, ohne seine Verbindung zu ändern, einfach mit dem einen Ende am axialen Protoplasma, mit dem anderen am protoplasmatischen Wandbelag fortgleitend.

Wenn wir aber solche Vorgänge massenhaft alle Tage im lebenden Protoplasma der'Zelle sehen können, so ist es sehr wohl möglich, dass innerhalb der durch die Centren festgelegten statischen, trajektoriellen Plasmastruktur die Chromosomen, welche doch schließlich auch Protoplasmaklumpen sind, in den Fäden als Leitbahnen sich fortbewcgen, ohne dass wir uns diese Bewegungen mechanisch erklären können.

Absichtlich gehe ich auf die Natur dieser Fäden, nämlich ob es durch Zug deformirte Waben oder aus dem Mitom gesponnene Fäden sind, nicht ein. Für die Frage nach der trajektoriellen Struktur kommen diese Dinge meiner Meinung nach in erster Linie nicht in Betracht. Eine Verquickung derselben mit der Frage der Trajektorien erscheint mir eher schädlich als nützlich, so wichtig ich auch die Plasmastrukturfrage an und für sich halte. Es ist offenbar nur ein Zufall, dass nach Meves diejenigen Autoren, welche bisher den sogenannten Centralkörpertheorien anhängeı, zugleich sämmtlich der Wabentheorie des Cytoplasmas in geringerem oder höherem Grade zugethan sind.

Die statisch trajektorielle Natur der Plasmastrahlungen gilt nur für die Mesophase, dem Höhepunkt der Mitose, während alle vorher- 
gehenden Stadien nur dem allmählichen Aufbau dieser statischen Struktur dienen und die nachfolgenden Stadien die statischen Trajektorien wieder abbrechen und auflösen, so dass. wir es vor und nach der Mesophase mit dynamischen Vorgängen zu thun haben.

Endlich weise ich für diesmal noch kurz darauf hin, dass organische Trajektorien ohne Spannung kaum denkbar sind, woraus folgt, dass sich der Nachweis von protoplasmatischen Trajektorien vielfach decken wird mit der Lehre M. Heinenhain's von den gespannten Protoplasmafäden. Es wird nöthig sein, in dieser Hinsicht weitere Vergleiche anzustellen. 